\title{
A global inversion-symmetry-broken phase inside the pseudogap region of $\mathrm{YBa}_{2} \mathrm{Cu}_{3} \mathrm{O}_{y}$
}

\author{
L. Zhao ${ }^{1,2}$, C. A. Belvin ${ }^{3}$, R. Liang ${ }^{4,5}$, D. A. Bonn ${ }^{4,5}$, W. N. Hardy ${ }^{4,5}$, N. P. Armitage ${ }^{6}$ and D. Hsieh ${ }^{1,2 \star}$
}

The phase diagram of cuprate high-temperature superconductors features an enigmatic pseudogap region that is characterized by a partial suppression of low-energy electronic excitations ${ }^{1}$. Polarized neutron diffraction ${ }^{2-4}$, Nernst effect $^{5}$, terahertz polarimetry ${ }^{6}$ and ultrasound measurements ${ }^{7}$ on $\mathrm{YBa}_{2} \mathrm{Cu}_{3} \mathrm{O}_{y}$ suggest that the pseudogap onset below a temperature $T^{*}$ coincides with a bona fide thermodynamic phase transition that breaks time-reversal, four-fold rotation and mirror symmetries respectively. However, the full point group above and below $T^{*}$ has not been resolved and the fate of this transition as $T^{*}$ approaches the superconducting critical temperature $T_{c}$ is poorly understood. Here we reveal the point group of $\mathrm{YBa}_{2} \mathrm{Cu}_{3} \mathrm{O}_{y}$ inside its pseudogap and neighbouring regions using high-sensitivity linear and second-harmonic optical anisotropy measurements. We show that spatial inversion and two-fold rotational symmetries are broken below $T^{*}$ while mirror symmetries perpendicular to the $\mathrm{Cu}-\mathrm{O}$ plane are absent at all temperatures. This transition occurs over a wide doping range and persists inside the superconducting dome, with no detectable coupling to either charge ordering or superconductivity. These results suggest that the pseudogap region coincides with an odd-parity order that does not arise from a competing Fermi surface instability and exhibits a quantum phase transition inside the superconducting dome.

The crystal system and point group of a material are encoded in the structure of its second- and higher-rank optical susceptibility tensors ${ }^{8}$, which can be determined from the anisotropy of its linear and nonlinear optical responses. The second-harmonic (SH) response is particularly sensitive to the presence of global inversion symmetry because unlike the linear electric-dipole susceptibility tensor $\chi_{i j}^{\mathrm{ED}}$, which is allowed in all crystal systems, the SH electricdipole susceptibility tensor $\chi_{i j k}^{\mathrm{ED}}$ vanishes in centrosymmetric point groups, leaving typically the far weaker electric-quadrupole term $\chi_{i j k l}^{\mathrm{EQ}}$ as the primary bulk radiation source ${ }^{9,10}$. For these reasons, it has been proposed that optical SH generation may be an effective probe of inversion symmetry breaking in the cuprates ${ }^{11}$.

To fully resolve the spatial symmetries underlying the pseudogap and its neighbouring regions, we performed both linear and $\mathrm{SH}$ optical rotational anisotropy (RA) measurements on de-twinned single crystals of $\mathrm{YBa}_{2} \mathrm{Cu}_{3} \mathrm{O}_{y}$ as a function of oxygen content and temperature. The RA measurements track variations in the intensities of light reflected at the fundamental $\left(I^{\omega}\right)$ and $\mathrm{SH}$ frequencies $\left(I^{2 \omega}\right)$ of an obliquely incident laser beam, which is resonant with the $\mathrm{O} 2 p$ to $\mathrm{Cu} 3 d$ charge transfer energy $(\hbar \omega=1.5 \mathrm{eV})$, as the scattering plane is rotated about the $c$ axis (Fig. 1a). The use of a recently developed rotating optical grating-based technique ${ }^{12}$ made it possible to perform full $360^{\circ}$ sweeps of the scattering plane angle $(\varphi)$ over large temperature ranges while keeping the incident beam spot $(\sim 100 \mu \mathrm{m})$ fixed to the same location on the crystal to within a few micrometres.

We first simulate the RA patterns expected from hole-doped $\mathrm{YBa}_{2} \mathrm{Cu}_{3} \mathrm{O}_{y}(y>6)$ based on its reported orthorhombic $\mathrm{mmm}$ crystallographic point group ${ }^{13}$, which is inversion symmetric and consists of three generators $m_{\mathrm{ac}}, m_{\mathrm{bc}}$ and $m_{\mathrm{ab}}$ that denote mirror symmetries across the $a-c, b-c$ and $a-b$ planes. Unlike its tetragonal parent $(y=6)$ compound $^{14}$, the point group of holedoped $\mathrm{YBa}_{2} \mathrm{Cu}_{3} \mathrm{O}_{y}$ endows the crystal only with two-fold $\left(C_{2}\right)$ rather than four-fold $\left(C_{4}\right)$ rotational symmetry about the $c$ axis because the excess oxygen atoms form chains that run along the $b$ axis (Fig. 1a). Figure $1 \mathrm{~b}$ shows representative linear and SH RA patterns computed in the electric-dipole $I^{\omega}(\varphi) \propto\left|\hat{e}_{i}^{\omega}(\varphi) \chi_{i j}^{\mathrm{ED}} \hat{e}_{j, 0}^{\omega}(\varphi)\right|^{2} I_{0}$ and electricquadrupole $I^{2 \omega}(\varphi) \propto\left|\hat{e}_{i}^{2 \omega}(\varphi) \chi_{i j k l}^{\mathrm{EQ}} \hat{e}_{j, 0}^{\omega}(\varphi) \partial_{k} \hat{e}_{l, 0}^{\omega}(\varphi)\right|^{2} I_{0}^{2}$ approximations respectively, where $I_{0}$ is the incident beam intensity and both the incident (in) and reflected (out) polarizations $\hat{e}_{0}$ and $\hat{e}$ are selected to be perpendicular $\left(S_{\text {in }}-S_{\text {out }}\right)$ to the scattering plane (see Supplementary Section 1 for other polarization geometries). The orthorhombic crystal system is identifiable by an ovular $I^{\omega}(\varphi)$ pattern with maxima and minima aligned strictly along the $a$ and $b$ axes ${ }^{15}$ while the $\mathrm{mmm}$ point group is identifiable by a four-lobed $I^{2 \omega}(\varphi)$ pattern that is mirror symmetric about the $a$ and $b$ axes.

Figure 2 shows linear and $\mathrm{SH} \mathrm{RA}$ data from $\mathrm{YBa}_{2} \mathrm{Cu}_{3} \mathrm{O}_{y}$ with hole-doping levels $(p)$ of $0.125\left(y=6.67\right.$; underdoped; $\left.T_{c}=65 \mathrm{~K}\right)$, $0.135\left(y=6.75\right.$; underdoped; $\left.T_{c}=75 \mathrm{~K}\right), 0.165(y=6.92$; optimal doped; $\left.T_{\mathrm{c}}=92 \mathrm{~K}\right)$ and $0.190\left(y=7.0 ;\right.$ overdoped; $\left.T_{\mathrm{c}}=86 \mathrm{~K}\right)$ measured at room temperature $\left(T>T^{*}\right)$ in $S_{\text {in }}-S_{\text {out }}$ geometry (see Supplementary Section 2 for all other geometries). In the linear RA data, we see that the anisotropy $\left(\chi_{x x}^{\mathrm{ED}}-\chi_{y y}^{\mathrm{ED}}\right)$ becomes more pronounced with hole doping as expected due to the filling of $\mathrm{Cu}-\mathrm{O}$ chains. However, in contrast to Fig. 1b, the intensity maxima and minima are rotated away from the $a$ and $b$ axes, indicating an absence of $m_{\mathrm{ac}}$ and $m_{\mathrm{bc}}$ symmetries consistent with a monoclinic distortion. In fact, by using the structure of $\chi_{i j}^{\mathrm{ED}}$ for a monoclinic crystal system in the expression for $I^{\omega}(\varphi)$, excellent fits to the data are obtained (Fig. 2). In the SH RA data, we also find clear violations of the $\mathrm{mmm}$ point group symmetries at all doping levels. In particular, the alternation in lobe magnitude as a function of $\varphi$ and the rotation of the lobe bisectors away from the $a$ and $b$ axes indicate an absence of $m_{\mathrm{ac}}$ and $m_{\mathrm{bc}}$ symmetries consistent with the linear RA data. We find excellent agreement of the data with an electricquadrupole-induced $\mathrm{SH}$ response from the centrosymmetric $2 / \mathrm{m}$ monoclinic point group (Fig. 2), which consists of two generators 2 and $m$ that denote $C_{2}$ and $m_{\mathrm{ab}}$ symmetries. On the contrary, fits

\footnotetext{
${ }^{1}$ Department of Physics, California Institute of Technology, Pasadena, California 91125, USA. ${ }^{2}$ Institute for Quantum Information and Matter, California Institute of Technology, Pasadena, California 91125, USA. ${ }^{3}$ Wellesley College, Wellesley, Massachusetts 02481, USA. ${ }^{4}$ Department of Physics and Astronomy, University of British Columbia, Vancouver, British Columbia V6T 1Z1, Canada. ${ }^{5}$ Canadian Institute for Advanced Research, Toronto, Ontario M5G 1Z8, Canada. 'Institute of Quantum Matter, Department of Physics and Astronomy, The Johns Hopkins University, Baltimore, Maryland 21218, USA. *e-mail: dhsieh@caltech.edu
} 


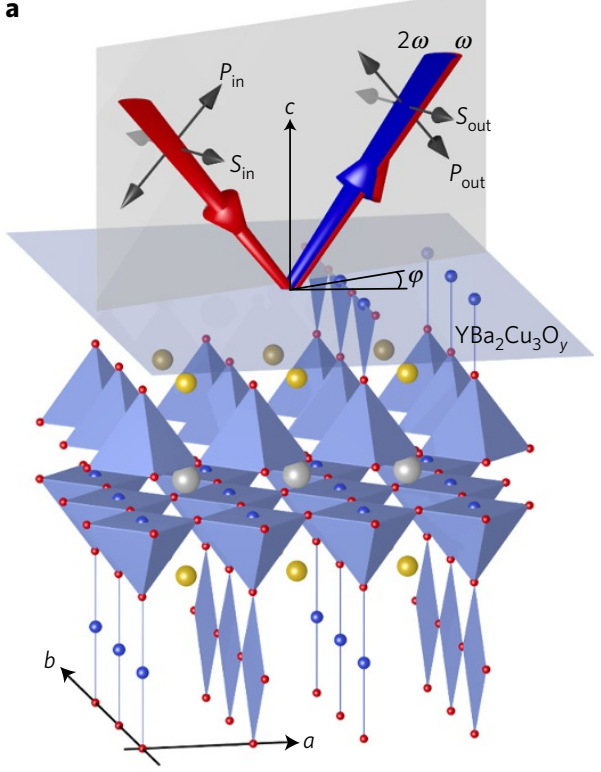

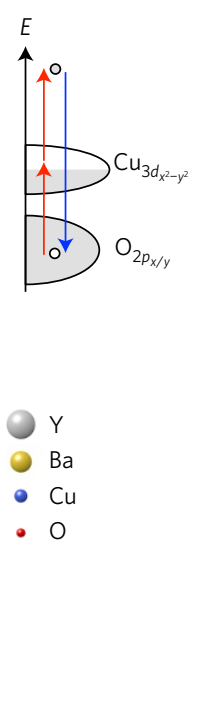

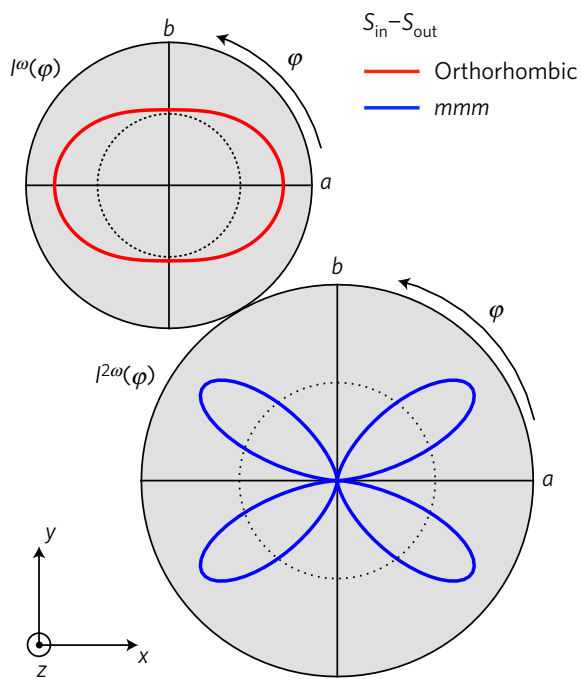

Figure 1 | Layout of optical anisotropy experiment and predicted response of $\mathrm{YBa}_{2} \mathrm{Cu}_{3} \mathrm{O}_{y}$. $\mathbf{a}$, The intensity of light reflected at the fundamental $\left(I^{\omega}\right)$ and $\mathrm{SH}$ $\left(2^{2 \omega}\right)$ frequencies of an obliquely incident beam is measured as a function of the angle $(\varphi)$ between the scattering plane and the $a-c$ plane of $\mathrm{YBa}_{2} \mathrm{Cu}_{3} \mathrm{O}_{y}$. The $\mathrm{Cu}-\mathrm{O}$ chains run along the $b$ axis. The incident photon energy $(1.5 \mathrm{eV})$ is resonant with the $\mathrm{O} 2 p$ to $\mathrm{Cu} 3 d$ charge transfer transition (inset). The polarization of the incident (in) and reflected (out) beams can be selected to lie either parallel $(P$ ) or perpendicular (S) to the scattering plane. $\mathbf{b}$, Rotational anisotropy of the electric-dipole-induced linear response and electric-quadrupole-induced SH response simulated for the orthorhombic crystal system and the $\mathrm{mmm}$ point group respectively in $S_{\text {in }}-S_{\text {out }}$ geometry. The crystallographic $a$ and $b$ axes are aligned respectively along the laboratory $x$ and $y$ axes.
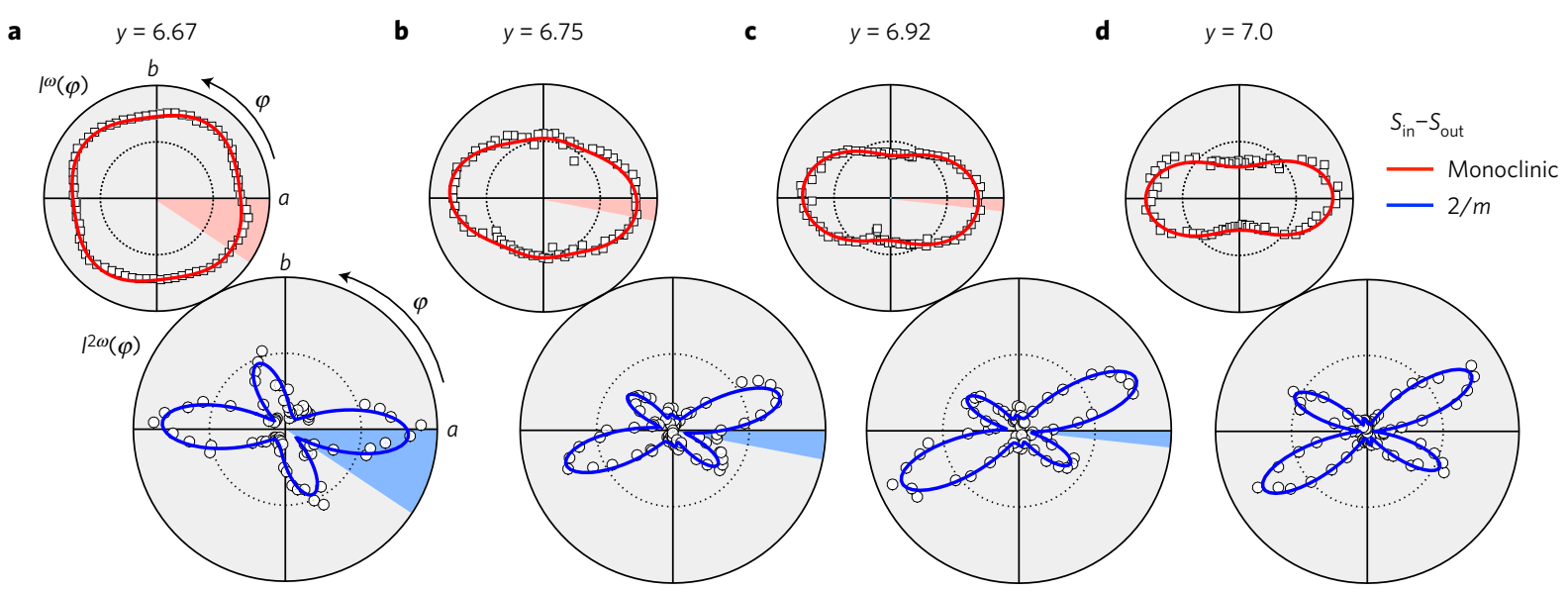

Figure 2 | Crystal system and point group of $\mathrm{YBa}_{2} \mathrm{Cu}_{3} \mathrm{O}_{y}$ above $T^{*}$. a-d, Polar plots of $I^{\omega}(\varphi)$ and $I^{2 \omega}(\varphi)$ measured at $T=295 \mathrm{~K}$ in $S_{\text {in }}-S_{\text {out }}$ geometry from $\mathrm{YBa}_{2} \mathrm{Cu}_{3} \mathrm{O}_{y}$ with $y=6.67(\mathbf{a}), 6.75(\mathbf{b}), 6.92(\mathbf{c})$ and $7.0(\mathbf{d})$. The $l^{\omega}(\varphi)$ and $l^{2 \omega}(\varphi)$ data sets, which have intensity error bars of approximately $\pm 1 \%$ and $\pm 10 \%$ respectively, are each plotted on the same intensity scale with a scaling ratio of $l^{\omega}(\varphi): I^{2 \omega}(\varphi) \approx 1: 3 \times 10^{-11}$. The former are fitted to the electric-dipole-induced linear response of the monoclinic crystal system (red lines) and the latter are fitted to the electric-quadrupole-induced SH response of the $2 / \mathrm{m}$ point group (blue lines). The angular deviations of the maxima of $I^{\omega}(\varphi)$ and lobe bisectors of $I^{2 \omega}(\varphi)$ away from the $a$ axis are shaded red and blue respectively for clarity.

to electric-dipole-induced SH from the non-centrosymmetric 2 and $m$ monoclinic point groups, as well as to magnetic-dipole-induced ${ }^{11}$ or surface electric-dipole-induced $\mathrm{SH}$ from the $2 / \mathrm{m}$ point group, do not adequately describe the data (see Supplementary Section 3).

The degree of monoclinicity, which can be qualitatively tracked via the angular deviation of the intensity maximum (lobe bisector) in the linear (SH) RA data away from the $a$ axis, decreases monotonically between $y=6.67$ and $y=7$ as shown in Fig. 2. This suggests that the absence of $m_{\mathrm{ac}}$ and $m_{\mathrm{bc}}$ symmetries at all measured temperatures above $T^{*}$ (see Supplementary Section 4) originates from vacancy-induced monoclinic distortions of the oxygen sublattice, which are also known to be present in $\mathrm{La}_{2-x} \mathrm{Sr}_{x} \mathrm{CuO}_{4}$ (ref. 16). This interpretation is further supported by recent SH RA measurements performed near the charge transfer resonance of layered perovskite iridates $^{9,10}$, which revealed subtle oxygen sublattice distortions that are difficult to resolve using diffraction-based probes.

Having established the point group of hole-doped $\mathrm{YBa}_{2} \mathrm{Cu}_{3} \mathrm{O}_{y}$ above $T^{*}$, we proceed to search for changes in symmetry across the strange metal to pseudogap boundary. Previous infrared conductivity experiments ${ }^{17}$ have shown that optical transition rates at frequencies well above the pseudogap energy scale $(\hbar \omega \gtrsim 0.3 \mathrm{eV})$ do not exhibit any measurable temperature dependence across $T^{*}$. As a corollary, any temperature-dependent change in magnitude of $\chi_{i j}^{\mathrm{ED}}$ or $\chi_{i j k l}^{\mathrm{EQ}}$ at the frequencies used in this study $(\hbar \omega=1.5 \mathrm{eV}$ 
a
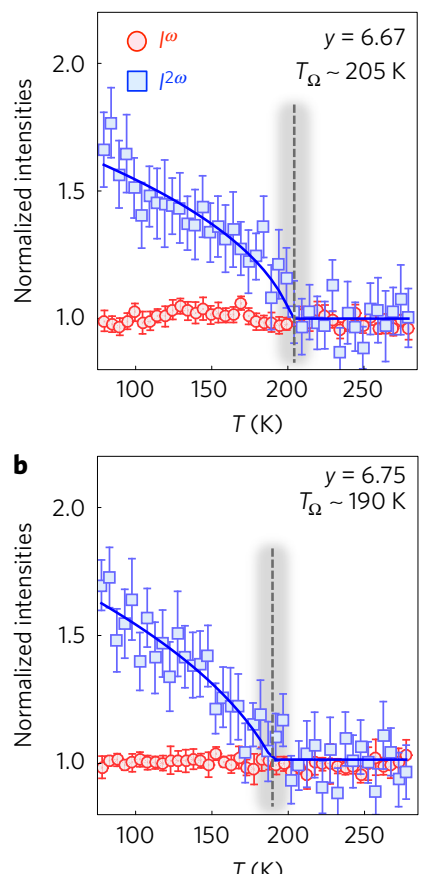

c

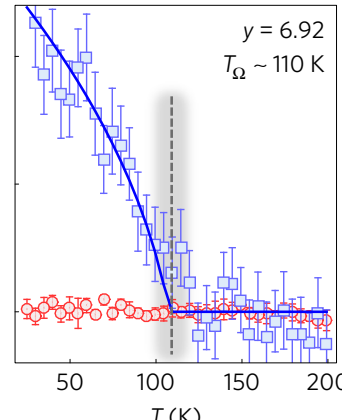

d

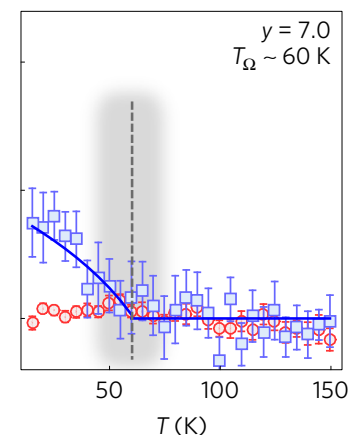

e

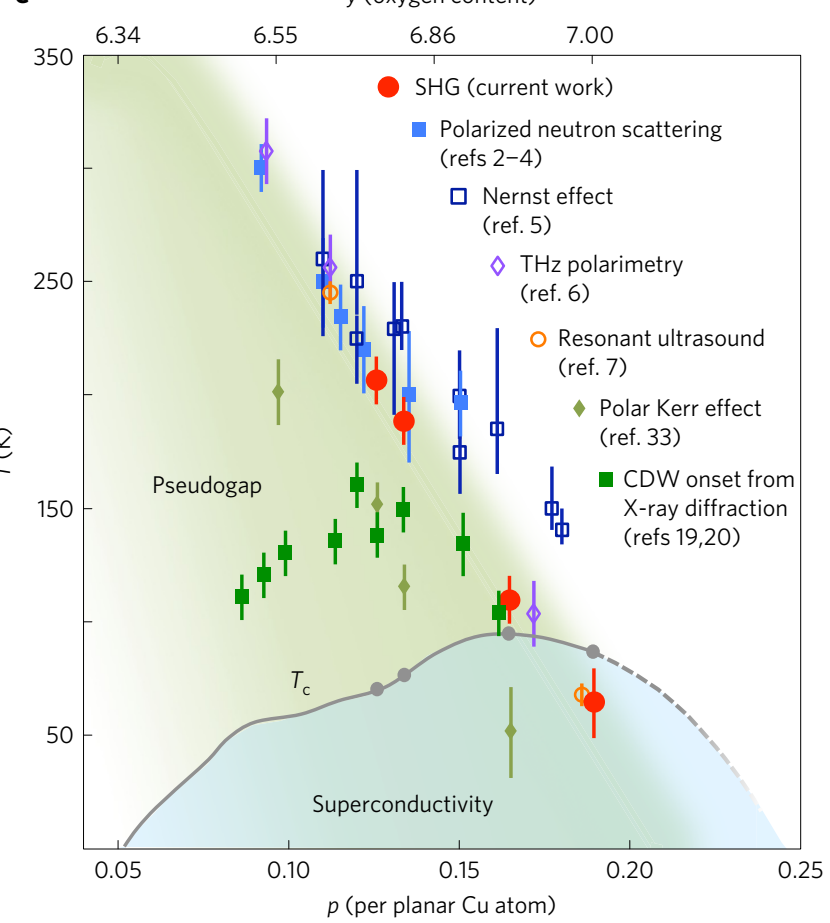

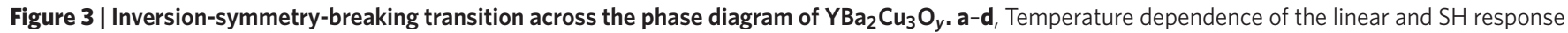

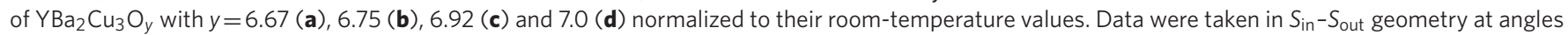
$\varphi$ corresponding to the smaller SH lobe maxima at $T=295 \mathrm{~K}$ (see Fig. 2). The error bars represent the standard deviation of the intensity over 60 independent measurements. Blue curves overlaid on the data are guides to the eye. The inversion-symmetry-breaking transition temperatures $T_{\Omega}$ determined from the $\mathrm{SH}$ response are marked by the dashed lines. The width of the shaded grey intervals represents the uncertainty in $T_{\Omega}$. e, Temperature versus doping phase diagram of $\mathrm{YBa}_{2} \mathrm{Cu}_{3} \mathrm{O}_{y}$. The values of $T_{\Omega}$ (red circles) coincide with the onset of the pseudogap as defined by spin-polarized neutron

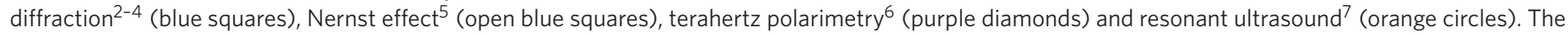
onset of a polar Kerr effect ${ }^{33}$ (green diamonds) and short-range charge order as determined by $X$-ray diffraction ${ }^{19,20}$ (green squares) occur at temperatures below $T^{*}$. Error bars denote the uncertainty in the onset temperatures. The superconducting transition temperatures of the samples used in our work are denoted by grey circles.

and $2 \hbar \omega=3 \mathrm{eV}$ ) should be correspondingly weak. Figure $3 \mathrm{a}-\mathrm{d}$ shows the temperature dependences of both the linear and $\mathrm{SH}$ intensities measured at a fixed value of $\varphi$ in $S_{\text {in }}-S_{\text {out }}$ geometry. For all four doping levels studied in Fig. 2, we observe no change in the linear response as a function of temperature as expected. Surprisingly however, all of the $\mathrm{SH}$ responses exhibit a significant order-parameter-like upturn below a doping-dependent critical temperature $T_{\Omega}$. This dichotomy between the linear and $\mathrm{SH}$ responses can naturally be reconciled if bulk inversion symmetry is broken below $T_{\Omega}$, which would turn on a new and stronger source of electric-dipole-induced $\mathrm{SH}$ radiation on top of the already existing electric-quadrupole contribution.

By plotting the doping dependence of $T_{\Omega}$ atop the phase diagram of $\mathrm{YBa}_{2} \mathrm{Cu}_{3} \mathrm{O}_{y}$ (Fig. 3e), we find that the observed onset of inversion symmetry breaking coincides very well with the pseudogap phase boundary $T^{*}$ defined by spin-polarized neutron diffraction ${ }^{2-4}$, Nernst anisotropy ${ }^{5}$, terahertz polarimetry ${ }^{6}$ and resonant ultrasound ${ }^{7}$ measurements in the optimal and underdoped regions, suggesting a common underlying mechanism. Moreover, we detect an onset of inversion symmetry breaking even inside the superconducting dome in the overdoped region, which may imply a quantum phase transition slightly beyond $p=0.20$ near where the pseudogap energy scale has also been extrapolated to vanish ${ }^{18}$. Interestingly, the temperature dependence of the SH intensity shows no measurable anomalies after crossing either the superconducting or the charge density wave ordering ${ }^{19,20}$ temperatures (Fig. 3e) and does not rapidly diminish at lower temperatures like the Nernst anisotropy ${ }^{5}$, which may be caused by charge density wave ordering ${ }^{21}$. This shows that unlike the superconducting and charge-ordered phases, which are competing Fermi surface instabilities ${ }^{22}$, the observed inversion-symmetrybroken phase is independent of and coexistent with both of them.

To determine which symmetries in addition to inversion are removed from the $2 / m$ point group below the pseudogap temperature, we performed a comparative study of the SH RA data measured above and below $T_{\Omega}$. Figure 4a shows RA patterns measured at $295 \mathrm{~K}$ and $30 \mathrm{~K}$ in $S_{\text {in }}-S_{\text {out }}$ geometry for the optimal doped sample $\left(T_{\Omega} \sim 110 \mathrm{~K}\right)$, which is representative of all other doping levels studied (see Supplementary Section 5). Below $T_{\Omega}$ we see that the $\mathrm{SH}$ intensity is enhanced in a $\varphi$-dependent manner that preserves the two-fold rotational symmetry of the pattern, which ostensibly implies that $C_{2}$ is preserved in the crystal. However, this interpretation can be ruled out because the inferred above electric-dipole-induced $\mathrm{SH}$ radiation is strictly forbidden by symmetry in $S_{\text {in }}-S_{\text {out }}$ geometry for any point group (non-magnetic or magnetic) that contains $C_{2}$ (see Supplementary Section 3). The only alternative interpretation is that the order parameter below $T_{\Omega}$ breaks $C_{2}$ symmetry, but is obscured in the RA patterns because of spatial averaging over domains of two degenerate orientations of the order parameter that are related by $180^{\circ}$ rotation about the $c$ axis. This would imply a characteristic domain length scale that is much smaller than our laser spot size and consistently explains the absence of any signatures of $C_{2}$ breaking below $T^{*}$ in Nernst effect ${ }^{5}$ and terahertz polarimetry ${ }^{6}$ data as well as the need for multiple magnetic domains to refine the polarized neutron diffraction data $^{2-4,23}$, which are all 
a

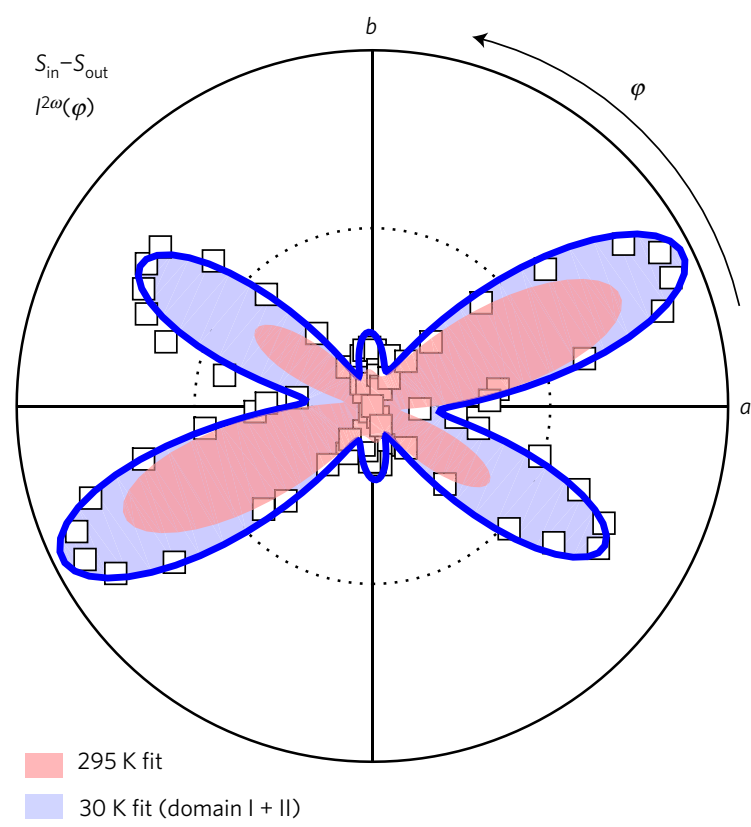

b

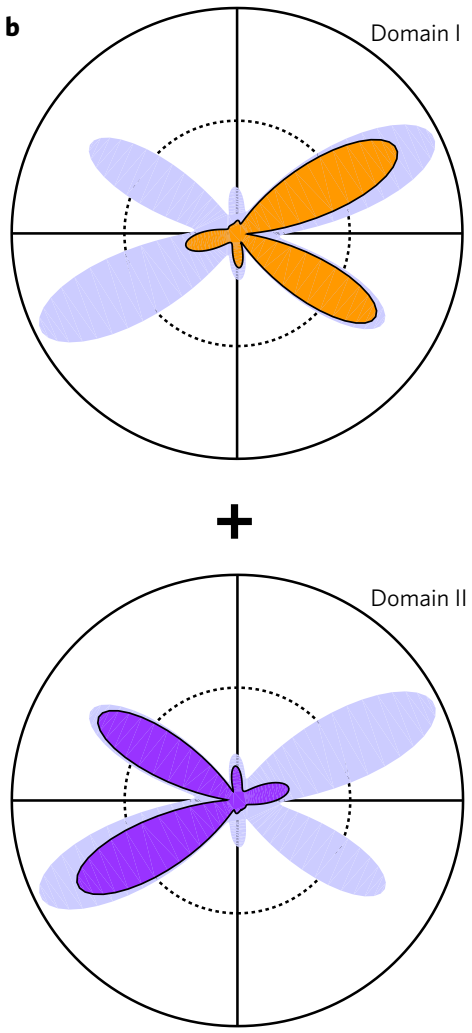

Figure 4 | Point group symmetry of $\mathrm{YBa}_{2} \mathrm{Cu}_{3} \mathrm{O}_{y}$ below $T^{*}$. a, Polar plot of $R^{2 \omega}(\varphi)$ measured at $T=30 \mathrm{~K}$ in $\mathrm{S}_{\text {in }}-\mathrm{S}_{\text {out }}$ geometry from $\mathrm{YBa}_{2} \mathrm{Cu}_{3} \mathrm{O}_{6.92}(\mathrm{squares})$. The intensity error bars are approximately $\pm 10 \%$. The blue curve is a best fit to the average of two $180^{\circ}$-rotated domains with $2^{\prime} / \mathrm{m}$ point group symmetry as described in the text. The fit to the $T=295 \mathrm{~K}$ data (pink shaded area) reproduced from Fig. $2 \mathrm{c}$ is overlaid for comparison. $\mathbf{b}$, Decomposition of the fit to the $T=30 \mathrm{~K}$ data (shaded blue area) into its individual domain contributions (shaded orange and purple areas).

integrated over even larger areas of the crystal compared with our measurements.

The set of all non-centrosymmetric subgroups of $2 / m$ that do not contain $C_{2}$ consists of the two independent magnetic point groups $2^{\prime} / m$ and $m 1^{\prime}$ and their associated magnetic and non-magnetic subgroups (see Supplementary Section 6), where the generators $2^{\prime}, m$ and $1^{\prime}$ denote $C_{2}$ combined with time-reversal, $m_{\mathrm{ab}}$ and the identity operation combined with time-reversal respectively. Using the two-domain $(\alpha=1,2)$ averaged expression $I^{2 \omega}(\varphi) \propto$ $\sum_{\alpha=1,2}\left|\hat{e}_{i}^{2 \omega}(\varphi) \chi_{i j k l}^{\mathrm{EQ}} \hat{e}_{j, 0}^{\omega}(\varphi) \partial_{k} \hat{e}_{l, 0}^{\omega}(\varphi)+\hat{e}_{i}^{2 \omega}(\varphi) \chi_{i j k, \alpha}^{\mathrm{ED}} \hat{e}_{j, 0}^{\omega}(\varphi) \hat{e}_{k, 0}^{\omega}(\varphi)\right|^{2} I_{0}^{2}$, we were able to reproduce all features of the low-temperature $\mathrm{SH}$ RA data, including the small peaks around the $b$ axis (Fig. 4a), by applying the structure of $\chi_{i j k, \alpha}^{\mathrm{ED}}$ for either the $2^{\prime} / m$ or $m 1^{\prime}$ point group. The decomposition of the fit into its two single-domain components is shown in Fig. $4 \mathrm{~b}$ to explicitly illustrate the loss of $C_{2}$. An equally good fit to the data can naturally be achieved using any magnetic or non-magnetic subgroup of $2^{\prime} / m$ or $m 1^{\prime}$ because they necessarily allow the same or more non-zero independent $\chi_{i j k, \alpha}^{\mathrm{ED}}$ tensor elements. Therefore, while further removal of symmetry elements from $2^{\prime} / m$ or $m 1^{\prime}$ is not necessary to explain the low-temperature data, it cannot be completely ruled out.

In conclusion, our results show that the pseudogap region in $\mathrm{YBa}_{2} \mathrm{Cu}_{3} \mathrm{O}_{y}$ is bounded by a line of phase transitions associated with the loss of global inversion and $C_{2}$ symmetries. Although previous terahertz polarimetry measurements on hole-doped $\mathrm{YBa}_{2} \mathrm{Cu}_{3} \mathrm{O}_{y}$ thin films reported the onset of a linear dichroic response near $T^{*}$ that breaks $m_{\mathrm{ac}}$ and $m_{\mathrm{bc}}$ symmetries ${ }^{6}$, we find that these symmetries are already broken in the crystallographic structure above $T^{*}$ and are thus necessarily absent in any tensor response that turns on below $T^{*}$. The low symmetry of the point group (probably $2^{\prime} / m$ or $m 1^{\prime}$ ) underlying the pseudogap region cannot be explained by stripe ${ }^{24}$ or nematic ${ }^{5}$ type orders alone, which have also been reported to develop below $T^{*}$. Instead it suggests the presence of an odd-parity magnetic order parameter, which is consistent with theoretical proposals involving a ferroic ordering of current loops circulating within the $\mathrm{Cu}-\mathrm{O}$ octahedra ${ }^{11,25-27}$, local $\mathrm{Cu}$-site magnetic quadrupoles $^{28}$, O-site moments ${ }^{29}$ or magneto-electric multipoles generated dynamically through spin-phonon coupling ${ }^{30}$. Regardless of microscopic origin, our results suggest that this order undergoes a quantum phase transition inside the superconducting dome slightly beyond optimal doping, which may be responsible for the enhanced $T_{c}$ and quasiparticle mass ${ }^{31}$ observed in its vicinity. Interestingly, a similar odd-parity magnetic phase has also recently been found in the pseudogap region of a $5 d$ transition metal analogue of the cuprates ${ }^{32}$, which hints at a possibly more robust connection between the pseudogap and this unusual form of broken symmetry.

\section{Methods}

Methods, including statements of data availability and any associated accession codes and references, are available in the online version of this paper.

Received 30 June 2016; accepted 13 October 2016; published online 21 November 2016

\section{References}

1. Keimer, B., Kivelson, S. A., Norman, M. R., Uchida, S. \& Zaanen, J. From quantum matter to high-temperature superconductivity in copper oxides. Nature 518, 179-186 (2015).

2. Fauqué, B. et al. Magnetic order in the pseudogap phase of high- $T_{\mathrm{C}}$ superconductors. Phys. Rev. Lett. 96, 197001 (2006).

3. Mook, H. A., Sidis, Y., Fauqué, B., Balédent, V. \& Bourges, P. Observation of magnetic order in a superconducting $\mathrm{YBa}_{2} \mathrm{Cu}_{3} \mathrm{O}_{6.6}$ single crystal using polarized neutron scattering. Phys. Rev. B 78, 20506(R) (2008). 
4. Mangin-Thro, L., Sidis, Y., Wildes, A. \& Bourges, P. Intra-unit-cell magnetic correlations near optimal doping in $\mathrm{YBa}_{2} \mathrm{Cu}_{3} \mathrm{O}_{6.85}$. Nat. Commun. 6, 7705 (2015).

5. Daou, R. et al. Broken rotational symmetry in the pseudogap phase of a high- $T_{\mathrm{c}}$ superconductor. Nature 463, 519-522 (2010).

6. Lubashevsky, Y., Pan, L., Kirzhner, T., Koren, G. \& Armitage, N. P. Optical birefringence and dichroism of cuprate superconductors in the $\mathrm{THz}$ regime. Phys. Rev. Lett. 112, 147001 (2014).

7. Shekhter, A. et al. Bounding the pseudogap with a line of phase transitions in $\mathrm{YBa}_{2} \mathrm{Cu}_{3} \mathrm{O}_{6+\delta}$. Nature 498, 75-77 (2013).

8. Boyd, R. W. Nonlinear Optics (Academic, 2003).

9. Torchinsky, D. H. et al. Structural distortion-induced magnetoelastic locking in $\mathrm{Sr}_{2} \mathrm{IrO}_{4}$ revealed through nonlinear optical harmonic generation. Phys. Rev. Lett. 114, 96404 (2015).

10. Hogan, T. et al. Structural investigation of the bilayer iridate $\mathrm{Sr}_{3} \mathrm{Ir}_{2} \mathrm{O}_{7}$. Phys Rev. B 93, 134110 (2016).

11. Simon, M. E. \& Varma, C. M. Symmetry considerations for the detection of second-harmonic generation in cuprates in the pseudogap phase. Phys. Rev. B 67, 54511 (2003).

12. Torchinsky, D. H., Chu, H., Qi, T., Cao, G. \& Hsieh, D. A low temperature nonlinear optical rotational anisotropy spectrometer for the determination of crystallographic and electronic symmetries. Rev. Sci. Instrum. 85, 83102 (2014).

13. Shaked, H. Crystal Structures of the High- $T_{\mathrm{c}}$ Superconducting Copper-Oxides (Elsevier, 1994).

14. Jorgensen, J. D. et al. Structural properties of oxygen-deficient $\mathrm{YBa}_{2} \mathrm{Cu}_{3} \mathrm{O}_{7-\delta}$. Phys. Rev. B 41, 1863-1877 (1990).

15. Schmid, H. et al. Polarized light and $X$-ray precession study of the ferroelastic domains of $\mathrm{YBa}_{2} \mathrm{Cu}_{3} \mathrm{O}_{7-\delta}$. Z. Phys. B 72, 305-322 (1988).

16. Reehuis, M. et al. Crystal structure and high-field magnetism of $\mathrm{La}_{2} \mathrm{CuO}_{4}$. Phys. Rev. B 73, 144513 (2006).

17. Lee, Y. S. et al. Electrodynamics of the nodal metal state in weakly doped high- $\mathrm{T}_{\mathrm{c}}$ cuprates. Phys. Rev. B 72, 54529 (2005).

18. Tallon, J. L. \& Loram, J. W. The doping dependence of $T^{*}-$ what is the real high- $\mathrm{T}_{\mathrm{c}}$ phase diagram? Physica C 349, 53-68 (2001).

19. Blanco-Canosa, S. et al. Resonant $x$-ray scattering study of charge-density wave correlations in $\mathrm{YBa}_{2} \mathrm{Cu}_{3} \mathrm{O}_{6+x}$. Phys. Rev. B 90, 54513 (2014).

20. Hücker, M. et al. Competing charge, spin, and superconducting orders in underdoped $\mathrm{YBa}_{2} \mathrm{Cu}_{3} \mathrm{O}_{y}$. Phys. Rev. B 90, 54514 (2014).

21. Cyr-Choinière, O. et al. Two types of nematicity in the phase diagram of the cuprate superconductor $\mathrm{YBa}_{2} \mathrm{Cu}_{3} \mathrm{O}_{y}$. Phys. Rev. B 92, 224502 (2015).

22. Chang, J. et al. Direct observation of competition between superconductivity and charge density wave order in $\mathrm{YBa}_{2} \mathrm{Cu}_{3} \mathrm{O}_{6.67}$. Nat. Phys. 8, 871-876 (2012)

23. Li, Y. et al. Unusual magnetic order in the pseudogap region of the superconductor $\mathrm{HgBa}_{2} \mathrm{CuO}_{4+\delta}$. Nature 455, 372-375 (2008).

24. Parker, C. V. et al. Fluctuating stripes at the onset of the pseudogap in the high- $\mathrm{T}_{\mathrm{c}}$ superconductor $\mathrm{Bi}_{2} \mathrm{Sr}_{2} \mathrm{CaCu}_{2} \mathrm{O}_{8+x}$. Nature 468, 677-680 (2010).
25. Varma, C. M. Non-Fermi-liquid states and pairing instability of a general model of copper oxide metals. Phys. Rev. B 55, 14554-14580 (1997).

26. Simon, M. E. \& Varma, C. M. Detection and implications of a time-reversal breaking state in underdoped cuprates. Phys. Rev. Lett. 89, 247003 (2002).

27. Yakovenko, V. M. Tilted loop currents in cuprate superconductors. Physica B 460, 159-164 (2015)

28. Lovesey, S. W., Khalyavin, D. D. \& Staub, U. Ferro-type order of magneto-electric quadrupoles as an order-parameter for the pseudo-gap phase of a cuprate superconductor. J. Phys. Condens. Matter 27, 292201 (2015).

29. Moskvin, A. S. Pseudogap phase in cuprates: oxygen orbital moments instead of circulating currents. JETP Lett. 96, 385-390 (2013)

30. Fechner, M., Fierz, M. J. A., Thöle, F., Staub, U. \& Spaldin, N. A. Quasistatic magnetoelectric multipoles as order parameter for pseudogap phase in cuprate superconductors. Phys. Rev. B 93, 174419 (2016).

31. Ramshaw, B. J. et al. Quasiparticle mass enhancement approaching optimal doping in a high- $\mathrm{T}_{c}$ superconductor. Science 348, 317-320 (2015).

32. Zhao, L. et al. Evidence of an odd-parity hidden order in a spin-orbit coupled correlated iridate. Nat. Phys. 12, 32-36 (2016).

33. Xia, J. et al. Polar Kerr-effect measurements of the high-temperature $\mathrm{YBa}_{2} \mathrm{Cu}_{3} \mathrm{O}_{6+x}$ superconductor: evidence for broken symmetry near the pseudogap temperature. Phys. Rev. Lett. 100, 127002 (2008).

\section{Acknowledgements}

We thank D. N. Basov, P. Bourges, B. Keimer, S. A. Kivelson, P. A. Lee, J. W. Lynn, J. Orenstein, S. Raghu, B. Ramshaw, C. Varma and N.-C. Yeh for valuable discussions. This work was supported by ARO Grant W911NF-13-1-0059. Instrumentation for the RA measurements was partially supported by ARO DURIP Award W911NF-13-1-0293. D.H. acknowledges funding provided by the Institute for Quantum Information and Matter, an NSF Physics Frontiers Center (PHY-1125565) with support of the Gordon and Betty Moore Foundation through Grant GBMF1250. N.P.A. acknowledges support from ARO Grant W911NF-15-1-0560. Work at the University of British Columbia was supported by the Canadian Institute for Advanced Research and the Natural Science and Engineering Research Council.

\section{Author contributions}

L.Z., D.H. and N.P.A. planned the experiment. L.Z. and C.A.B. performed the RA measurements and N.P.A. determined the crystal alignment. L.Z., D.H. and N.P.A. analysed the data. R.L., D.A.B. and W.N.H. prepared and characterized the samples. L.Z. and D.H. wrote the manuscript.

\section{Additional information}

Supplementary information is available in the online version of the paper. Reprints and permissions information is available online at www.nature.com/reprints.

Correspondence and requests for materials should be addressed to D.H.

\section{Competing financial interests}

The authors declare no competing financial interests. 


\section{Methods}

Material growth. $\mathrm{YBa}_{2} \mathrm{Cu}_{3} \mathrm{O}_{y}$ single crystals were grown in non-reactive $\mathrm{BaZrO}_{3}$ crucibles using a self-flux technique. The $\mathrm{Cu}-\mathrm{O}$-chain oxygen content was set to $y=6.67,6.75,6.92$ and 7.0 by annealing in a flowing $\mathrm{O}_{2} / \mathrm{N}_{2}$ mixture and homogenized by further annealing in a sealed quartz ampoule, together with ceramic at the same oxygen content. The absolute oxygen content $(y)$ is accurate to \pm 0.01 based on iodometric titration. The crystals used in our experiments were de-twinned and aligned to high accuracy by X-ray Laue diffraction.

Optical RA measurements. The RA measurements were performed using a rotating optical grating-based technique ${ }^{12}$ with ultrashort ( $\sim 80 \mathrm{fs}$ ) optical pulses produced from a regeneratively amplified Ti:sapphire laser operating at a $10 \mathrm{kHz}$ repetition rate. The angle of incidence was $\sim 30^{\circ}$ and the incident fluence was maintained below $1 \mathrm{~mJ} \mathrm{~cm}^{-2}$ to ensure no laser-induced changes to the samples (see Supplementary Section 7). Alignment of the optical axis to the crystallographic $c$ axis was determined to better than $0.1^{\circ}$ accuracy as described in Supplementary Section 8. Reflected fundamental $(\lambda=800 \mathrm{~nm})$ and SH light $(\lambda=400 \mathrm{~nm})$ were collected using photodiodes and photomultiplier tubes respectively. Crystals were sealed in a dry environment during transportation and immediately pumped down to pressures $<5 \times 10^{-6}$ torr for measurements.

Fitting procedure. The high-temperature $\left(T>T_{\Omega}\right)$ linear optical anisotropy data were fitted to the expression $I^{\omega}(\varphi)=A\left|\hat{e}_{i}^{\omega}(\varphi) \chi_{i j}^{\mathrm{ED}} \hat{e}_{j, 0}^{\omega}(\varphi)\right|^{2} I_{0}$ and the high- $\left(T>T_{\Omega}\right)$ and low-temperature $\left(T<T_{\Omega}\right)$ SH anisotropy data were fitted to the expressions $I^{2 \omega}(\varphi)=\left|A \hat{e}_{i}^{2 \omega}(\varphi) \chi_{i j k l}^{\mathrm{EQ}} \hat{e}_{j, 0}^{\omega}(\varphi) \partial_{k} \hat{e}_{l, 0}^{\omega}(\varphi)\right|^{2} I_{0}^{2}$ and
$I^{2 \omega}(\varphi)=\left(|A|^{2} / 2\right) \sum_{\alpha=1,2}\left|\hat{e}_{i}^{\omega \omega}(\varphi) \chi_{i j k l}^{\mathrm{EQ}} \hat{e}_{j, 0}^{\omega}(\varphi) \partial_{k} \hat{e}_{i, 0}^{\omega}(\varphi)+\hat{e}_{i}^{2 \omega}(\varphi) \chi_{i j k, \alpha}^{\mathrm{ED}} \hat{e}_{i, 0}^{\omega}(\varphi) \hat{e}_{k, 0}^{\omega}(\varphi)\right|^{2} I_{0}^{2}$ respectively. Here $A$ is a constant determined by the experimental geometry, $\hat{e}_{0}$ and $\hat{e}$ are the polarizations of the incoming and outgoing light in the frame of the crystal, $\chi_{i j}^{\mathrm{ED}}$ is the linear electric-dipole susceptibility tensor, $\chi_{i j k l}^{\mathrm{EQ}}$ is the $\mathrm{SH}$ electric-quadrupole susceptibility tensor and $\chi_{i j k, \alpha}^{\mathrm{ED}}$ is the SH electric-dipole susceptibility tensor. The $\alpha=1$ and $\alpha=2$ versions of $\chi_{i j k, \alpha}^{\mathrm{ED}}$ are related by $180^{\circ}$ rotation about the $c$ axis. The non-zero independent elements of these tensors in the frame of the crystal are determined by applying the monoclinic crystal symmetries to $\chi_{i j}^{\mathrm{ED}}$ and the appropriate point group symmetries $(2 / m$ for $\chi_{i j k l}^{\mathrm{EQ}}$ and either $2 / m$ or $m 1^{\prime}$ for $\left.\chi_{i j k, \alpha}^{\mathrm{ED}}\right)$ and degenerate SH permutation symmetries to $\chi_{i j k l}^{\mathrm{EQ}}$ and $\chi_{i j k, \alpha}^{\mathrm{ED}}$. These operations reduce $\chi_{i j}^{\mathrm{ED}}$ to 5 non-zero independent elements $(x x, x z, y y, z x, z z), \chi_{i j k l}^{\mathrm{EQ}}$ to 28 non-zero independent elements $(x x x x, y y y y, z z z z, x x y y=x y y x, y y x x=y x x y, x y x y, y x y x, x x z z=x z z x, z z x x=$ $z x x z, x z x z, z x z x, y y z z=y z z y, z z y y=z y y z, y z y z, z y z y, x z y y=x y y z, x y z y, y x z y=$ $y y z x, y x y z=y z y x, y y x z=y z x y, z x y y=z y y x, z y x y, z x x x, x x z x, x x x z=$ $x z x x, x z z z, z z x z, z z z x=z x z z)$, and $\chi_{i j k, \alpha}^{\mathrm{ED}}$ to 10 non-zero independent elements $(x x x, x y x=x x y, x y y, x z z, y x x, y y x=y x y, y y y, y z z, z z x=z x z, z z y=z y z)$. Values of $R^{2}>0.95$ were achieved for all fits shown. However, the fitted values of the susceptibility tensor elements are not unique. Therefore, the fits serve only to address the qualitative question of whether a certain point group can or cannot reproduce the data. They are not intended to convey any quantitative information about the magnitudes of various tensor elements.

Data availability. The data that support the plots within this paper and other findings of this study are available from the corresponding author on request. 